\title{
Due voci per un dizionario di retorica
}

\author{
Sandro Briosi
}

\section{Metafora}

Il primo tentativo di definire la natura e le funzioni della metafora si trova nella Poetica di Aristotele che scrive: "La metafora consiste nel dare a una cosa il nome di un'altra." Nel caso di "il prato ride" viene attribuito al prato un elemento che "appartiene" all'uomo. Non è chiaro se questo rapporto di appartenenza sia per Aristotele un rapporto naturale, originario. Egli non contrappone in effetti l'uso figurato del linguaggio ad un uso "corretto" ma ad un uso "corrente" o "normale" ("kùrion"). In ogni caso la sostituzione non muta profondamente la natura dell'oggetto indicato ed essa ha per lui una funzione essenzialmente estetica, di abbellimento, simile a quella svolta da altri usi "anormali" del linguaggio. Essi danno al discorso un sapore di "stranezza" che però non dev'esser mai eccessivo, tale da nascondere la chiarezza e la riconoscibilità del termine "proprio" sostituito.

Il trasferimento può avvenire, per Aristotele, in quattro modi: dal genere alla specie ("Qui è ferma la mia nave": "esser fermo" è il genere, "essere all'ancora" è una specie, uno dei modi dell'esser fermo); dalla specie al genere ("Diecimila azioni": un numero specifico al posto del generico "molte"); da una specie ad un'altra ("spegnere la vita" al posto di "troncare"; ambedue sono speci di "togliere"); per analogia. Per mostrare l'astrattezza di questa classificazione basterebbe chiedersi: "spegnere" era già una "specie" del genere "togliere" prima che la metafora accostasse i due termini? Non si potrebbe dire che il genere di "spegnere" è "far finire" (la luce, la vita), oppure "rendere oscuro" (un luogo, e la vita intesa come visione, conoscenza)? La metafora per analogia gioca invece per Aristotele su quattro elementi, legati dallo schema di una proporzione: $\mathrm{A}: \mathrm{B}=\mathrm{C}: \mathrm{D}$. Questa spiegazione verrà ripresa da molte teorie moderne e per ciò vale la pena di osservare, contro di essa, quanto segue: che cosa ci impedisce di considerare la vecchiaia e la sera come speci del genere "fine," spiegando così la tipica metafora analogica come metafora da specie a specie?; e di contro, il caso di "essere fermo" ("estànai") detto invece del termine proprio "essere all'ancora" ("ormèin") non si può spiegare, invece che come metafora da specie a specie, come espressione che sottintende la proporzione estànai: uomo = ormèin: nave?

Il fatto è che, malgrado le numerose intuizioni che la contraddicono, la teoria aristotelica resta fondata sul rifiuto di riconoscere la forza di innovazione, di creazione di speci e generi nuovi che abita la metafora. Anche la retorica latina considera la nostra figura come uno degli "ornamenti" del discorso. Per 
Quintiliano, a questa funzione di abbellimento rispondono anzitutto gli artifici (gli arcaismi, i neologismi, la "gradazione," l" "accumulazione," l' "esagerazione" e la "diminuzione") che non cambiano il significato delle parole, a differenza di ciò che avviene con la metafora e gli altri "tropi." E qui dobbiamo subito notare come la distinzione sia forzata ed astratta. In "è un bandito," frase data come esempio di "esagerazione," riconosciamo infatti una metafora poiché il termine non è usato nel senso "proprio." Questa difficoltà di Quintiliano a distinguere i casi in cui c'è o con c'è cambiamento di significato si spiega d'altronde considerando il criterio fondamentale che regola per lui l'uso degli ornamenti e dei tropi: il criterio dell'essere aderenti alla realtà, dell'usare la decorazione in modo "appropriato" rispetto all'oggetto decorato. Anche la similitudine (e la metafora non è che una "similitudine abbreviata") non dev'essere per lui "falsa" ma "adatta ai soggetti" (VIII; 3, 76); ed altrove (VIII; 2, 10) Quintiliano arriva a scrivere che "anche le parole felicemente traslate sogliono esser chiamate proprie." Dove in fondo si rivela la contraddizione che abita l'intiero progetto della retorica classica: il progetto di una classificazione degli artifici utili ad apparir spontanei e naturali nel quale la metafora deve essere allora un uso . . . appropriato dell'improprietà. L'esagerazione, l'inverosimiglianza va evitata; ma va evitata anche, nell' uso dei tropi, l'eccessiva "umiltà" e prevedibilità (VIII; 6, 14): il criterio del gusto si sostituisce così a quello della verosimiglianza. Il buon gusto coincide allora col buon senso e la metafora, realtà posta tra le ragioni della Ragione e quelle della Bellezza, viene ancora una volta spogliata della tensione conoscitiva ed innovativa che la caratterizza quando è una metafora "viva." Eppure i termini tra cui nasce questa tensione sono ben presenti: "la metafora è stata escogitata per commuovere gli animi" (VIII; 6, 19) ed anche perché "esprime meglio qualcosa" (VIII; 6, 6); ma la "commozione" diventa subito un mezzo non per dire qualcosa di nuovo bensì per meglio aderire alla realtà esterna. Lo stesso sentimento, come l'opera dell'abbellimento, deve essere "appropriato" trovando la propria giustificazione nell'indubitabile oggettività del mondo.

Le contraddizioni rilevabili nella retorica greca e latina scompaiono nel Medioevo. Rispetto all'assolutezza delle verità della Rivelazione cristiana la funzione conoscitiva del linguaggio e dei suoi processi viene messa ancor più in ombra. La verità del significato delle cose è garantita dal suo scaturire da Dio e non può per ciò esser mai totalmente conoscibile. Ogni sospetto di differenza tra metafora (figura "motivata," necessaria ed abitata dal "sentimento") e allegoria (figura non motivata e "razionale") viene a cadere, il lettore coglie la metafora come qualcosa che non ha nulla di "proprio" ed è "sprovvista di ogni frangia inquietante" (Zumthor). Così Dante (nel Comivio e nella lettera a Cangrande della Scala) distingue tra senso letterale, allegorico, morale ed anagogico usando come esempio la frase biblica "In exitu Israel de Aegipto, domus Jacob de populo barbaro, facta est Iudaea sanctificatio eius, Israel potestas eius." Il senso allegorico è la redenzione operata 
da Cristo; il senso morale è la conversione dal peccato allo stato di grazia, il senso anagogico "l'uscita dell'anima santa dal servaggio di questa corruzione alla libertà della gloria eterna” (trad. di G. Vinay). Il senso più vicino all'esperienza, personale dello scrittore e del lettore, il senso morale, si trova posto entro una gerarchia che non gli lascia alcuna possibilità di introdurre un elemento di innovazione o di personale interpretazione nell'ordine terreno e divino della Verità. Ciò non significa naturalmente che in Dante l'allegoria cancelli ogni uso personale, metaforico del linguaggio: una volta si diceva con Croce che nella Divina Commedia non c'è solo allegoria ma anche "poesia." Significa solo che, là dove si verifica la "rivelazione personale" dell'immagine poetica, essa appare allo serittore come una forza contenuta non nello "spazio" che si apre tra il senso proprio e quello figurato (Genette) ma contenuta tutta nel senso letterale: "tantôt-scrive Zumthor-spécialement chez de grands poètes de la fin du Moyen age, le littéral confère au figuratif une telle force de credibilité que toute idée abstraite s'efface et que l'allégorie prend la valeur d'une signification personnelle."

Dopo l'epoca rinascimentale, che rimane legata alle concezioni aristoteliche, nell'età barocca la riflessione sulla metafora registra una svolta. Di essa può esser preso come esempio il più interessante teorizzatore dell" "ingegnosità" poetica, Emanuele Tesauro. La sua opera si colloca nel quadro della crisi della fiducia aristotelica nell'ordine razionale del mondo ed insieme dell'incapacità di sostituirla con la fede in un ordine invisibile ed assoluto. Nel Cannocchiale aristotelico la sorpresa, la "meraviglia" provocata dalla metafora diventano i mezzi per comunicare un significato nuovo: la metafora è una "enimmatica voce, oscuramente chiara e tacitamente parlante, per fare indovino l'ascoltatore." Ci pare, questa, una formula efficacissima nella sua capacità di cogliere e di accettare la contraddizione apparente fra la chiarezza della metafora e l" oscurità" che la circonda quando si tenti si "spiegarla," di parafrasarla; e tra la parola necessaria a realizzare la figura ed il carattere "silenzioso," cioè ancora non riducibile ad altri significati dati, del suo senso vero. E questo senso è una realtà estranea all'ordine del linguaggio costituito, è un senso inseparabile dall" "emozione" che suscita la metafora: essa infatti ci induce a trovare "dentr'una paroletta molti concetti, leggergli in parte nello scritto e il rimanente nel cuor di chi scrive." L'invenzione metaforica non è più qualcosa che abbellisce, rischiando se mal usata di falsarli, le "speci" e i "generi" delle cose; l'accostamento di oggetti lontani diventa anzi, in Tesauro, un procedimento che imita quello seguito dalla Natura e dalla stessa creazione divina. "Le prodigiose cadute dei fulmini (sono) formidabili arguzie e simboliche cifre della Natura," e le stesse invenzioni degli uomini sembrano scguire questa spinta verso la confusione dei generi e la creazione di generi e speci inedite: "Dragoni fischianti [. . . testuggini animate da" corpi umani [...] istrici, scorpioni [...] tutte ingegnose ma fiere metafore omicide."

Un'altra via seguita per rovesciare il rapporto di superiorità del senso 
proprio rispetto all "ornamento" metaforico è quella seguita da Vico ne la scienza nuova. Vico è il primo ad affermare che la metafora è la forma originaria del linguaggio. "I primi poeti dieder a' corpi l'essere di sostanze animate," egli scrive affermando così che le prime metafore erano in fondo degli errori i quali solo quando "si ritruovarono le voci che significavano forze astratte, ognuni comprendenti le loro spezic [...] (tai parlari) sono diventati trasporti." Anche la metonimia e la sineddoche sono nate come errori: il primo uomo che ha detto "poppa" per nave in realtà vedeva, all'orizzonte, apparir solo questa "parte" del "tutto." Resta aperto il problema di spiegare perché, una volta scoperto l'errore, le figure non siano scomparse dal linguaggio; ma ciò nulla toglie all'importanza di questo primo sforzo di spiegar la metafora come fenomeno legato ai concreti modi dell'esperienza percettiva e non ai processi di un gioco operato sulle categorie date che restano, esse, il luogo della verità. Come le prime favole erano "vere narrazioni" così è vera anche la metafora che può definirsi una "piccola favoletta." Dove è interessante notare come Vico allarghi il campo della metafora fino a potervi comprendere un intero testo: non solo la singola parola può esser usata in modo improprio e "vero" ma anche un intiero discorso, il discorso del poeta.

Con Les figures du discours (1818) di Pierre Fontanier si assiste al primo grande tentativo di riprendere e sistematizzare le definizioni della retorica classica. Importante è la sua spiegazione del "piacere" provocato dalla metafora, che nasce dalle "jdee accessorie" (in termini moderni si direbbe dalle "connotazioni") evocate dal termine metaforico e sulle quali si concentra l'attenzione del parlante. Interessante è anche la sua ripresa della classica distinzione tra figure "di pensiero" e figure "di parola." Della seconda categoria fan parte per esempio le figure "di costruzione" che sembrano riguardare il solo ordine delle parole e non il loro senso (l'anacoluto, l'ellissi ecc.); della prima fan parte la "deliberazione," la "concessione," il "parallelo," tutti modi del discorso che hanno a che fare, evidentemente, coi suoi contenuti. Da una parte ci sono dei puri giochi di parole, dall'altra delle forme che coincidono perfettamente coi modi di prodursi e di articolarsi dello stesso pensiero. Ora, parlando dei tropi "ad una" o "a più" parole, Fontanier è costretto a creare una categoria nuova, quella delle figure "miste o doppie": figure, in certo senso, di parola e di pensiero insieme tra le quali si colloca la metafora. Egli intuisce così la posizione "di frontiera" del parlar metaforico, la sua natura di discorso che "gioca" col linguaggio per esprimere un "pensiero" che senza quel gioco sarebbe rimasto incomunicabile.

Alla spiegazione classicistica come quella di Fontanier si oppongono naturalmente, nel corso dell'Ottocento, molte interpretazioni della metafora che, romanticamente, insistono molto di più sul carattere "passionale" di questo tropo e sulla sua funzione conoscitiva legata al fatto (affermato tra i primi da $\mathrm{Hegel}$ ) del carattere motivato, necessario, non sostituibile e non parafrasabile dell'immagine metaforica. La concezione romantica della metafora tuttavia tende spesso a confondere questa figura con l'uso "vivo" del linguaggio in 
generale o ad usar la metafora per spiegare ogni uso “intuitivo" delle parole. "In questo senso tutto il Romanticismo opera una rimetaforizzazione delle forme artistiche negando l'aspetto 'retorico' e 'istituzionale' del suo operare," scrive G. Conte citando come esempio Shelley, per il quale (come per Vico) "nell'infanzia della società ogni autore è necessariamente poeta [. . . l'abbondanza della lessicografia e le distinzioni della grammatica sono opera di età più tarda": e tra queste distinzioni, c'è da pensare, è compresa anche quella tra senso proprio e senso metaforico. Ancora alla fine del secolo A. Biese potrà affermare che "non possiamo liberarci, pensando, dal meccanismo dei trasferimenti metaforici; una storia della metafora non è solo una storia degli errori umani, è una storia dell'umanità." Tutti i concetti che guidano il pensiero filosofico e scientifico non sono che metafore d'uso; un "pensiero puro," fondato su una corrispondenza univoca tra cose e parole, non è neppure concepibile. Il "grado zero," questa realtà mutevole e storica, era per Fontanier una realtà eterna ed indubitabile; per i romantici, esso non è che un mito, un'illusione. Il problema su cui il pensiero moderno si è concentrato è proprio questo: il problema dei rapporti tra l'uso proprio dei codici semiotici dati e gli atti di "invenzione" di cui la metafora "viva" rimane un caso esemplare. Se da una parte è vero, infatti, che la metafora introduce nel discorso un modo nuovo e "creativo" di "mettere in prospettiva" le cose (Black), è anche vero che questa novità può darsi solo tramite una "deviazione" dalle norme semantiche costituite. Senza un sistema dato di tali regole non potrebbero esserci metafore. Ma se l'uomo non possedesse questa capacità di "sospendere la referenza" (Ricoeur) per far emergere l'orizzonte di senso che egli dà alle cose, ogni suo discorso assomiglierebbe a quello di un computer ed ogni suo dialogo ai dialoghi, assurdi per eccesso di "logicità," delle commedie di Ionesco. In forme nuove, il dibattito contemporaneo riproduce in parte l'opposizione tra il razionalismo aristotelico e classicista e l" idealismo" vichiano e romantico. Da una parte potremo collocare le spiegazioni linguistiche e semiotiche (del tipo di Eco o del "Gruppo $\mu$ "); dall'altra, per esempio, i tentativi di comprendere psicanaliticamente la metafora come espressione dell'inconscio o luogo di emersione del "desiderio." Più fruttifera appare perciò la via seguita dalle indagini linguistico-logiche volte a comprendere $\mathrm{i}$ rapporti tra la metafora ed $\mathrm{i}$ "modelli" della scienza (Turbayne); e quella segnata dalla fenomenologia (Ricoeur) in cui la metafora è vista come un modo di "donazione di senso" che si scava il suo posto tra le costrizioni dei sistemi semiotici vigenti, dei modi dati di conoscere, di organizzare la realtà.

\section{Metonimia}

La retorica classica basa la distinzione tra metafora, da una parte, e metonimia e sineddoche dall'altra sulla differenza tra un rapporto di somiglianza, che caratterizza il primo caso, ed un rapporto di "contiguità" che caratterizza il secondo. La metafora consiste nella sostituzione di un termine ad un altro 
resa possibile dalla qualità comune ad ambedue che li rende simili: "la sera della vita" sostituisce "la vecchiaia" in quanto c la vita c la vecchiaia sono la fine di qualcosa. Nella metonimia la sostituzione avviene tra due termini uniti non da una somiglianza ma da rapporti "oggettivi" tra essi, dei quali la retorica ha offerto classificazioni oni accurate ma senza riuscire, e non sarà un caso, a definire con precisione questo carattere di "oggettività" dei rapporti e la sua differenza rispetto ai rapporti di significazione in gioco nella metafora. Così Fontanier può solo scrivere che le metonimie consistono "nella designazione di un oggetto attraverso il nome di un altro oggetto che è come il primo un tutto assolutamante a parte ma che gli deve o a cui esso deve qualcosa o per la sua esistenza o per il suo modo di essere": senza fare in questo grandi passi avanti rispetto alla definizione classica di Quintiliano. La classificazione prevede casi cose "bere un bicchiere" che sostituisce il contenente al contenuto; "leggere Manzoni” sostituisce l'autore all opera: "seguire la Croce (=il Cristianesimo)" sostituisce il segno all'oggetto; in altri casi il percorso va dalla causa all'effetto ("il pugnale- =la morte - ci ha liberato di lui"), dall'effetto alla causa ("vendere morte" = vendere armi), dal nome astratto al nome concreto e viceversa; eccetera. Perché, infine, si abbia metonimia il rapporto non deve essere quello, materiale, della "parte per il tutto" altrimenti ci si trova di fronte ("la vela" per "la nave") ad una sineddoche.

La distinzione classica si ritrova in molte teorie moderne. Così M. Le Guern afferma che la metonimia "si situa al di fuori del fatto propriamente linguistico" giocando sulle caratteristiche degli oggetti indicati e non sul contenuto semantico delle parole. L'opposizione tra metafora e metonimia come omologa a quella tra somiglianza e contiguità è stata ripresa da Jakobson che vedeva nelle due figure l'espressione dei due modi fondamentali del discorso e del pensiero. Per lui la metafora riguarda l" "asse della selezione" e perciò metaforico è ogni discorso (soprattutto quello poetico) che tende ad essere "autoriflessivo," a far sì che il linguaggio agisca soprattutto su sè stesso; metonimico è invece l'uso del linguaggio trasparente, prosastico, "referenziale."

In modi non molto diversi il problema viene posto dalle più moderne teorie semantiche e semiotiche. Per il "gruppo $\mu$ " non è tanto pertinente la differenza tra il mondo dei significati e l'oggettività dei referenti quanto quella fra termini aventi un tratto in comune (è l'antico concetto della "somiglianza") e termini facenti parte di una totalità data. Nel primo caso l'elemento unificatore è "inglobato" tra i due elementi messi in gioco nella metafora: in "l'uomo è un lupo" esso è costituito dalla cattiveria, punto di "intersezione" tra il mondo degli uomini e quello dei lupi. Nel caso della metonimia l'elemento unificatore è "inglobante": in "Prendete il vostro Cesare" (= prendete il vostro volume del De bello gallico) la sostituzione opera/autore è resa possibile all'interno di una totalità definibile come "la vita, gli amori c le opere di Cesare." Ad un'analisi attenta tutti gli sforzi, antichi c moderni, di dar consistenza ai rigidi confini tra metonimia e metafora, da una parte, c ira metonimia e sineddoche dall'altra si rivelano tuttavia discutibili. Nel secon- 
do caso risulta infatti difficile continuare a credere alla distinguibilità netta tra le parti "materiali" degli oggetti e le relazioni "ideali" o "logiche" che si danno tra essi. Per "seguì la Croce," per esempio, non si può dire che la croce è una parte materiale dell'universo cristiano? E di contro, per "bere un bicchiere" non si può dire che tra contenente e contenuto si dia una relazione simbolica, istituita da una tradizione culturale? Bere da un bicchiere, infatti, non è la stessa cosa che bere (come si fa in solitudine) direttamente dalla bottiglia ... Nel primo caso (rapporto metonimia/metafora) un'osservazione fondamentale potrebbe esser la seguente: tra oggetti "contigui" nella realtà si creano spesso delle relazioni di "intersezione" che li rendono "simili." Così la croce, "parte" del Cristianesimo, avrà potuto diventar simbolo di quest'ultimo grazie alle "connotazioni" di sofferenza e passione che essa evocava e che un certo modo di vivere il cristianesimo avrà riconosciuto "simili" a quelle associate indissolubilmente a tale religione. Si tratterebbe allora di un caso di metonimia ... metaforica, mentre di metafora ... metonimica si potrebbe parlare per "l'uomo è un lupo" se si ipotizzasse, seguendo il Gruppo $\mu$, un universo "inglobante" degli esseri cattivi all'interno del quale avverrebbe la sostituzione.

Queste osservazioni dovrebbero autorizzarci non a rifiutare i confini tradizionali fra le tre "figure principe" della retorica, ma a sottolineare il loro carattere formale ed esteriore ed a ricordare come più importante di questi confini sia la distinzione tra figure "vive" (cfr. Ricoeur), basate su un senso nuovo, non compreso nel codice come componente denotativa o connotativa del senso della parola, e figure basate su tratti comuni ("inglobanti" od "inglobati") già codificati.

Tenendo presente questa distinzione fondamentale sarà allora legittimo interrogarsi sul ruolo stilistico od espressivo che la metonimia può svolgere. Su questa via ha mosso già qualche passo Le Guern ma molto resterebbe da fare. Si pensi per esempio alla funzione ironica che hanno certe figure come "due Verdi" detto per "duemila lire," dove l'ironia nasce dal contrasto tra lo scarso valore della moneta e le connotazioni positive associate al musicista, e dove ancora si potrebbe discutere se si tratti di metonimia o di sineddoche: il volto di Verdi può esser visto infatti come parte materiale del foglio da mille lire o come suo simbolo. Spesso, la metonimia mette in primo piano immagini che non hanno tratti "vivi" in comune con la totalità che esse sostituiscono ma che evocano suggestioni giustificate dal contesto in cui esse si ritrovano in forme diverse: è ciò che avviene in Racine, come ha osservato Le Guern, che usa spesso "il sangue" nel senso di "la stirpe." In altri casi il sostituente proietta sul sostituito connotazioni particolari ("la poltrona" per "il posto" dà al posto un senso di sicurezza e di comodità, "la cattedra" dà al lavoro del professore un senso di autorità morale . . .). Qualche volta la metonimia si usa semplicemente per eufonia (per evitare la ripetizione del termine proprio); provoca effetti paradossali ed iperbolici ("si è mangiato la casa"), con eventuali prolungamenti "barocchi" che gio- 
cano sul doppio senso ("c la casa gli è rimasta sullo stomaco"); introduce nel discorso un ammiccamento di gergo rivolto agli iniziati ("la panchina" nel senso dell'allenatore e dei tecnici della squadra di calcio, che siedono su un'apposita panchina durante le partite); c in molti altri casi, naturalmente, l'espressività della figura nasce dal senso vivo che circonda l'oggetto scelto a rappresentare il termine proprio, esattamente come nella metafora. Un ultima osservazione che riteniamo importante riguarda tutti quei casi che definiremmo "metonimie apparenti": frasi in cui non si verifica alcun processo né di donazione "viva" di senso né di sostituzione di termini resa possibile dall'esistenza di elementi "inglobanti" od "inglobati" dafi. Cosi l'espressione "questa è la luce" usata per indicare il pulsante che comanda l'accensione della lampadina ci pare vada interpretata come una semplice ellissi, ma nel senso di cancellazione non tanto di alcune parole (come Le Guern ritiene avvenga sempre nella metonimia) quanto di alcuni elementi reali o reali relazioni tra le cose. Né la produzione né l'interpretazione di tale frase implica infatti alcuno spostamento dell'attenzione dall'oggetto indicato (il fenomeno fisico della luce) al termine "proprio" (il pulsante), e l'espressione indica in modo "abbreviato" ma diretto una certa realtà esperienziale (l'oggetto-premendo-il-quale-la-luce-s'accende). Più in generale una comprensione di ciò che avviene nell'uso della metonimia, come di ogni altra figura, non può basarsi sulla sola considerazione del "contenuto semantico" dei termini, definibile per via semiotica e differenziale, non può ignorare l'importanza di ciò che Le Guern stesso chiama la "concreta rappresentazione del referente." Se consideriamo che della concreta rappresentazione del referente "bere" fa parte l'elemento "portare alle labbra" (che non si può ricavare da un "analisi puramente linguistica centrata per esempio sull'opposizione bere/mangiare), potremo addirittura avanzar l' ipotesi che questo esempio "da manuale," già citato, di metonimia sia in realtà un espressione non figurata. Lo stesso vale per locuzioni (secondo Le Guern metonimiche) del tipo "sorriso ironico": non è corretto dire che ironica è "propriamente" la persona e non il sorriso se è vero che l'aggettivo viene spesso riferito direttamente alla particolare forma del volto che accompagna gli atteggiamenti ironici. Un progresso nello studio della metonimia insomma non può verificarsi se non abbandonando ogni rigida opposizione tra contenuti semantici e realtà referenziale, concepiti gli uni e l'altra come entità date all'interno delle quali si verificano gli spostamenti metonimici. Le Guern situa la metafora sul piano della rcaltà linguistica c la metonimia su quello della referenza, rimproverando $A$. Henry di tenersi sempre su questo secondo piano. Ma in realtà tra il mondo dei "significati" e quello delle "cose" andrebbe meglio considerata la presenza di quella "concreta rappresentazione del referente" che non è una passiva reazione della coscienza ma un atto di positiva "donazione di senso." 


\section{BIBLIOGRAFIA}

Aristotele. Opere, Vol. 10. Retorica. Poetica. Tr. di A. Plebe e M. Valgimigli. Bari: Laterza, 1973.

Biese, Alfred. Die Philosophie der Metaphorischen in Grundlinien Dargestelt. Hamburg-Leipzig: L. Voss, 1893.

Black, Max. Models and Metaphors. Ithaca and London: Cornell UP, 1962 e ss.

Conte, Giuseppe. Introduzione al vol. AA.VV. Metafora. Milano: Feltrinelli, 1981.

Dante Alighieri. Convivio e Lettera a Cangrande. In Opere. A cura di L. Blasucci. Firenze: Sansoni, 1965.

Eco, Umberto. Traltato di semiotica generale. Milano: Bompiani, 1975.

Fontanier, Pierre. Les figures du discours. Paris: Flammarion, 1968.

Genette, Gerard. Figures. Paris: Seuil, 1966.

Groupe $\mu$ (J. Dubois, F. Edeline, J. M. Klinkenberg, Ph. Minguct, F. Pire, H. Trinon). Rhétorique générale. Paris: Larousse, 1970 (tr. it. Milano: Bompiani, 1980).

Henry, Albert. Métonymie et métaphore. Paris: Klincsieck, 1971.

Jakobson, Roman. Saggi di linguistica generale. Tr. it. Milano: Feltrinelli, 1966.

Le Guern, Michel. Sémantique de la métaphore et de la métonymie. Paris: Flammarion, 1978.

Quintiliano. L'Istitnzione oratoria. A cura di R. Faranda c P. Pecchiura. Torino: UTET, 1972. Ricocur, Paul. La métaphore vive. Paris: Seuil, 1975.

Tesauro, Emanuele. Il carnocchiale aristotelico. In Trattatisti e narratori del Seicento. A cura di E. Raimondi. Milano-Napoli: Ricciardi, 1960.

Turbayne, Colin M. The Myth of Metaphor. U of South Carolina P, 1970.

Vico, Giambattista. La Scienza mova. A cura di F. Nicolini. Bari: Laterza, 1910-16 (3 voll.). Zumthor, Paul. Essais de poétique médievale. Paris: Seuil, 1971. 